\title{
Stationary states and phase diagram for a model of the Gunn effect under realistic boundary conditions
}

\author{
G. Gomila and J. M. Rubí \\ Department de Física Fonamental, Universitat de Barcelona, Diagonal 647, 08028 Barcelona, Spain \\ I. R. Cantalapiedra \\ Departament de Física Aplicada, Universitat Politécnica de Catalunya, Gregorio Marañón 44, 08028 Barcelona, Spain \\ L. L. Bonilla \\ Escuela Politécnica Superior, Universidad Carlos III de Madrid, Butarque 15, 28911 Leganés, Spain
}

(Received 13 March 1997)

\begin{abstract}
A general formulation of boundary conditions for semiconductor-metal contacts follows from a phenomenological procedure sketched here. The resulting boundary conditions, which incorporate only physically well-defined parameters, are used to study the classical unipolar drift-diffusion model for the Gunn effect. The analysis of its stationary solutions reveals the presence of bistability and hysteresis for a certain range of contact parameters. Several types of Gunn effect are predicted to occur in the model, when no stable stationary solution exists, depending on the value of the parameters of the injecting contact appearing in the boundary condition. In this way, the critical role played by contacts in the Gunn effect is clearly established. [S1063-651X(97)04608-4]

PACS number(s): 05.45.+b, 72.20.Ht, 85.30.Fg, 05.70.Ln
\end{abstract}

\section{INTRODUCTION}

The Gunn effect is a ubiquitous phenomenon in many semiconductor samples presenting negative differential resistance and subject to voltage bias conditions [1-4]. In a nutshell, the negative differential resistance makes possible the existence of a variety of pulses and wave fronts, which may be stabilized by the bias condition. Then a periodic shedding of waves by the injecting contact results in periodic oscillation of the current through an external circuit, which constitutes the signature of the Gunn effect. Although there is a vast literature on this topic, different basic questions concerning the Gunn effect remain poorly understood. Paramount among these, there are the questions concerning the correct boundary conditions and, given these, how to describe all the stages of the Gunn oscillation. The lack of a precise formulation of the boundary conditions imposed by contacts on semiconductors and of a simple analytic treatment to analyze the Gunn oscillations, has not allowed clarification of the role played by contacts in the Gunn effect. It is worth noting that clarifying this point would open, for instance, the possibility of extracting information about the contacts from an analysis of the Gunn oscillations themselves, a subject of considerable interest for applied researchers.

Recently, progress has been made toward answering these two questions reasonably. On the one hand, ideas from irreversible thermodynamics [5] have been used to derive satisfactory boundary conditions for metal-semiconductor and other contacts in a general way [6-8]. Previously the usual boundary conditions used in drift-diffusion semiconductor models were (i) periodic [9], (ii) charge neutrality [10], (iii) fixed field $[3,4]$, and (iv) control current-field characteristics of the contact [11], plus phenomenological assumptions such as the "contact length" [3]. As boundary conditions (b.c.'s) for a semiconductor presenting the Gunn effect, these conditions rank from clearly wrong (no current oscillation appears if the b.c.'s are periodic) to unsatisfactory because of their ad hoc character. Thus, even when numerical simulations display the Gunn effect, the question is usually raised of whether these results describe a real physical system where different contacts are present. In this paper, we shall present a simple derivation of appropriate b.c.'s for an ideal metalsemiconductor (MS) contact, and use them to analyze the Gunn effect in Kroemer's model for bulk $n$-type GaAs. Our description makes it clear which part of the derivation follows from general principles, and which part includes input from the physics of contacts.

Concerning asymptotic descriptions of the Gunn effect which delve deeper than just numerical simulations of driftdiffusion models, some progress has been made recently [12-15]. A detailed treatment of this topic can be found in Ref. [16].

The rest of the paper is as follows. In Sec. II we present our derivation of b.c.'s for ideal MS contacts, and briefly discuss some other possibilities. Kroemer's model and its stationary solutions for these b.c.'s are analyzed in Sec. III. It is found that bistability between stationary solutions is possible for certain bias ranges depending on the values of certain dimensionless contact parameters $i_{0}$ and $\alpha_{0}$, which are a combination of its effective density of states, barrier height, Richardson's constant, doping, and temperature. Different types of Gunn effect, namely, charge monopoles (moving charge accumulation and depletion layers) and charge dipoles (high- and low-field solitary waves), are predicted to appear depending on these contact parameters, when no stable stationary solution exists. In Sec. V we discuss our results, whereas the Appendix is devoted to technical matters related to the main text. 


\section{BOUNDARY CONDITIONS}

The aim of this section is to present a systematic procedure to derive b.c.'s at semiconductor contacts, established in previous works [6-8]. As a general rule, the method applies for nondegenerate semiconductors under moderate temperatures, that is, when thermionic emission is the dominant transport process at the contact. Hence several contacts of interest, like ideal and nonideal metal-semiconductor (MS) contacts or any type of heterojunction contact, can be modeled. Depending on the material parameters, both limiting as well as Ohmic contact may then be described. It is worth noting that a precise modeling of this type of contact may help to clarify the role played by other types of contacts used in semiconductor systems, e.g., those in which thermionicfield or field-emission processes dominate [17-19], for which a precise description, in the sense of the present paper, is not yet available. For the sake of clarity, the method will be presented along with its application to the case of an ideal MS contact. Other contacts have been considered in previous papers [6-8].

Let us consider an ideal MS contact. Due to the presence of the contact, the magnitudes describing the physical properties of the system, e.g., electron density, electric field, electron energy, etc., may be discontinuous at that point. In addition, singular contributions localized at the contact itself, e.g., electron density at interface states (when they are present), may also occur. As a consequence, a given physical magnitude, $d(\widetilde{x}, \widetilde{t})$, can be decomposed as follows:

$$
d(\tilde{x}, \tilde{t})=d_{n}(\tilde{x}, \tilde{t}) \theta(\tilde{x})+d_{m}(\tilde{x}, \tilde{t}) \theta(-\tilde{x})+d_{s}(\tilde{t}) \delta(\tilde{x}),
$$

where $d_{n}, d_{m}$, and $d_{s}$ refer to the values in the semiconductor $(n)$, metal $(m)$, and surface $(s)$ parts , respectively (when no singular contribution is present, $d_{s}$ vanishes). Moreover, $\theta(\tilde{x})$ is Heaviside's unit step function, and $\delta(\tilde{x})$ Dirac's delta function. They are introduced in order to represent the discontinuity across the contact and the singular contributions, respectively. In writing Eq. (1), a one-dimensional description of the system has been assumed, with the contact being located at $\tilde{x}=0$ and the metal (semiconductor) on its left (right). By means of this type of decomposition, b.c.'s can be systematically derived.

Our procedure consists of two steps: (a) the identification of the relevant magnitudes describing the transport processes through the contact; and (b) the derivation of precise laws describing such processes, which relate the relevant magnitudes at the contact and which constitute the desired b.c.'s. For the first step, use will be made of a phenomenological formulation of transport through semiconductor junctions [6] while for the second Shockley-Read-Hall (SRH) statistics [20,21] will be used.

Let us consider a given magnitude, $d(\tilde{x}, \widetilde{t})$, satisfying a standard balance equation of the form [5]

$$
\frac{\partial d(\tilde{x}, \tilde{t})}{\partial \tilde{t}}+\frac{\partial J_{d}(\tilde{x}, \tilde{t})}{\partial \tilde{x}}=\sigma_{d}(\tilde{x}, \tilde{t}),
$$

where $J_{d}(\tilde{x}, \tilde{t})$ and $\sigma_{d}(\tilde{x}, \tilde{t})$ refer to the current and net rate production associated with the magnitude $d$, respectively. It is not difficult to show that if similar balance equations were to be satisfied on each side of the junction and if surface fluxes only exist along the interface [which in a onedimensional description means $J_{d, s}(\widetilde{t})=0$ ], then the following balance equation should be satisfied at the contact [22]:

$$
\frac{\partial d_{s}(\tilde{t})}{\partial \widetilde{t}}+\left[J_{d, n}(0, \widetilde{t})-J_{d, m}(0, \tilde{t})\right]=\sigma_{d, s}(\widetilde{t})
$$

Now we can proceed to calculate the net rate of entropy production at the contact, which will allow us to identify the relevant magnitudes describing the transport processes through the contact. To begin with, we consider the balance equation for the total energy of the system. As this is a conserved quantity, we simply have

$$
\frac{\partial e_{s}(\widetilde{t})}{\partial \widetilde{t}}+\left[J_{e, n}(0, \tilde{t})-J_{e, m}(0, \tilde{t})\right]=0 .
$$

As for an ideal MS contact, no interface states are present, and hence no net charge or mass is accumulated at the contact, and the total energy at the contact coincides with the surface internal energy, $u_{s}=e_{s}$ [22]. Hence the balance of the internal energy is described directly through Eq. (4), or alternatively through

$$
\frac{\partial u_{s}(\widetilde{t})}{\partial \widetilde{t}}+\left[J_{u, n}(0, \widetilde{t})-J_{u, m}(0, \widetilde{t})\right]=\sigma_{u, s}(\widetilde{t})
$$

with

$\sigma_{u, s}(\widetilde{t})=\left[J_{u, n}(0, \widetilde{t})-J_{e, n}(0, \widetilde{t})\right]-\left[J_{u, m}(0, \widetilde{t})-J_{e, m}(0, \widetilde{t})\right]$

In the previous expression, we introduced explicitly the flux of internal energy (equivalent to the heat flux), which is in general different from the flux of total energy. Furthermore the Gibbs equation for an ideal contact is [22] $T d s_{s}=d u_{s}$ (no interface states are present), where $s_{s}$ is the surface entropy and $T$ the temperature. By assuming the contact to be in local equilibrium, one then has $T \partial s_{s}(\tilde{t}) / \partial \tilde{t}$ $=\partial u_{s}(\widetilde{t}) / \partial \widetilde{t}$, which, after using Eq. (5), gives rise to the balance equation for the entropy

$$
\frac{\partial s_{s}(\widetilde{t})}{\partial \widetilde{t}}+\left[J_{s, n}(0, \widetilde{t})-J_{s, m}(0, \widetilde{t})\right]=\sigma_{s, s}(\widetilde{t}),
$$

with the entropy production given by

$$
\begin{aligned}
\sigma_{s, s}(\widetilde{t})= & {\left[J_{s, n}(0, \widetilde{t})-\frac{1}{T} J_{e, n}(0, \widetilde{t})\right] } \\
& -\left[J_{s, m}(0, \widetilde{t})-\frac{1}{T} J_{e, m}(0, \widetilde{t})\right]
\end{aligned}
$$

A more explicit expression for $\sigma_{s, s}$ is obtained once the bulk expressions for the fluxes are introduced on the right-hand side of Eq. (7). These expressions can be found elsewhere $[5,6]$. One has 


$$
J_{s, a}(0, \widetilde{t})=\frac{1}{T} J_{e, a}(0, \widetilde{t})-\frac{1}{T} E_{F, a}(0, \widetilde{t}) J_{a}(0, \widetilde{t}), \quad a=m, n,
$$

where $E_{F, a}$ refers to the electron Fermi level (or chemical potential), and $J_{a}$ to the electron number density current. Substituting into Eq. (7), we simply have

$$
\begin{aligned}
\sigma_{s, s}(\widetilde{t}) & =-\frac{1}{T}\left[E_{F, n}(0, \widetilde{t}) J_{n}(0, \widetilde{t})-E_{F, m}(0, \widetilde{t}) J_{m}(0, \widetilde{t})\right] \\
& =-\frac{1}{T}\left[E_{F, n}(0, \widetilde{t})-E_{F, m}(0, \widetilde{t})\right] J_{n}(0, \widetilde{t}),
\end{aligned}
$$

where in the second line use has been made of the continuity of the electron number density current at an ideal MS contact [this continuity follows from the corresponding balance equation for the electron number density by imposing that no carriers are accumulated $\left[n_{s}(\widetilde{t})=0\right]$ nor created $\left[\sigma_{n, s}(\widetilde{t})\right.$ $=0]$ at the contact]. The final expression to be used in what follows is obtained by introducing the electron quasi-Fermi levels $F_{a}(0, \widetilde{t})=E_{F, a}(0, \widetilde{t})-e V_{a}(0, \widetilde{t})$. Here $V_{a}(0, \widetilde{t})$ is the electric potential (which is continuous through an abrupt junction) and $e>0$ is minus the charge of the electron. We then arrive at the desired expression

$$
\sigma_{s, s}(\widetilde{t})=-\frac{1}{T}\left[F_{n}(0, \widetilde{t})-F_{m}(0, \widetilde{t})\right] J_{n}(0, \widetilde{t}) .
$$

Equation (10) shows directly that the relevant magnitudes describing an ideal MS contact are the electron flux (electron current density divided by $e$ ) $J_{n}(0, \widetilde{t})$, and the discontinuity in the electron quasi-Fermi levels $\left[F_{n}(0, \widetilde{t})-F_{m}(0, \widetilde{t})\right]$, which plays the role of "thermodynamic force" [5]. Both flux and force vanish at equilibrium, and we assume (in accordance with the basic tenets of irreversible thermodynamics [5]) that there is a relation between them. When the fundamental relation between flux and force is specified, this relation is exactly the sought-after boundary condition at the contact.

The relation between $J_{n}(0, \widetilde{t})$ and $\left[F_{n}(0, \widetilde{t})-F_{m}(0, \widetilde{t})\right]$ should involve more information about the physics of the contact. First of all, let us note that the entropy production in Eq. (10) is formally equivalent to the expression corresponding to generation-recombination processes [23] (or, in general, to any activated process, such as unimolecular chemical reactions [5] or surface adsorption [24]), provided $J_{n}(0, \widetilde{t})$ is identified with the net rate of the process. From this comparison we then conclude that the transport through an ideal MS contact may be described as an elementary kinetic process of the form $q_{m} \rightleftharpoons q_{n}$, where $q_{n}$ and $q_{m}$ represent the carriers in the semiconductor and metal, respectively, with the net rate of the process being equal to $J_{n}(0, \widetilde{t})$. The kinetics of such a process can be described, for instance, by means of SRH statistics. As it is well known, this description relates the kinetic rate of the process, in our case $J_{n}(0, \widetilde{t})$, to the affinity, in our case the difference in quasi-Fermi levels, in agreement with our former general treatment. As shown in the Appendix we obtain the following relation between the net rate of the process, $J_{n}(0, \widetilde{t})$, and the jump of the quasi-Fermi level at the MS contact $\left[F_{n}(0, \widetilde{t})-F_{m}(0, \widetilde{t})\right]$ :

$$
J_{n}(0, \widetilde{t})=\lambda_{0} e^{-e \beta \phi_{b}^{0}\left(1-e^{\beta\left[F_{n}(0, \widetilde{t})-F_{m}(0, \widetilde{t})\right]}\right) .}
$$

Here $\lambda_{0}$ is a positive constant [see Eq. (A3) in the Appen$\operatorname{dix}]$, and $e \phi_{b}^{0}=E_{C}(0, \widetilde{t})-F_{m}(0, \widetilde{t})$ is the contact barrier height. $E_{C}(0, \widetilde{t})=E_{C}^{0}-e V_{n}(0, \widetilde{t})$ is the electron energy, with $E_{C}^{0}$ the bottom of the semiconductor conduction band and $V_{n}$ the electric potential at the semiconductor surface. Moreover, $\beta=(k T)^{-1}$, where $k$ is the Boltzmann constant. Equation (11) is the sought-after b.c. for the ideal MS contact. It can be written more explicitly by using the expression $\tilde{n}=N_{C} e^{\beta\left(F_{n}-E_{C}\right)}$, which holds for nondegenerate semiconductors, with $\tilde{n}$ being the semiconductor electron number density and $N_{C}$ the effective density of states. We then have

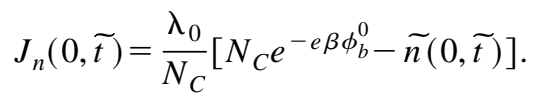

In the nondegenerate case, $\lambda_{0}$ depends only on $T$ (see the Appendix). This result ends the derivation of the b.c.'s for ideal MS contacts which we will use for the rest of this paper. It is worth emphasizing at this point that, as mentioned at the beginning of this section, the procedure we have sketched here for the ideal MS contact, can be applied to several other types of contacts. These include nonideal MS contacts with the presence of interface states [7] and unipolar or bipolar heterojunction contacts with or without interface states [7]. Moreover, by adding a few assumptions, one can handle nonabrupt contacts [8].

Note that for a MS contact located at $\tilde{x}=\widetilde{L}$, that is, with the metal [semiconductor] on the right- (left-) hand side of the contact, the corresponding b.c. is

$$
J_{n}(\widetilde{L}, \widetilde{t})=-\frac{\lambda_{L}}{N_{C}}\left[N_{C} e^{-e \beta \phi_{b}^{L}}-\tilde{n}(\widetilde{L}, \widetilde{t})\right] .
$$

We can compare our result, Eq. (12) for the ideal MS contact with the corresponding one reported in Ref. [25] (see also p. 261 of Ref. [26]). Then we can identify $\lambda_{i}=A_{i} T^{2} / e, i=0, \widetilde{L}$, where $A_{i}$ is the Richardson constant for the semiconductor in contact with the metal located at $i=0, \widetilde{L}$. Theoretically, $A_{i}$, and hence $\lambda_{i}$, would depend only on the given semiconductor but not on the metal [26]. However, in practice $A_{i}$ is taken as a phenomenological parameter, and it cannot depend only on the metal but also on the preparation procedures [27]. On the other hand, basic energetic arguments lead immediately to the following rule for the contact barrier height [26,28]: $\phi_{b}^{i}=\phi_{M}^{i}-\chi$. Here $\phi_{M}^{i}$ is the work function of the metal in the MS contact located at $\tilde{x}=i$, and $\chi$ is the semiconductor electron affinity. For covalent semiconductors, the validity of this rule has been put under question for the last five decades [17,18]. However, recently it was shown that even for this type of semiconductor, if accurate growth materials are used, good agreement is obtained with this simple rule $[29,30]$. Note that, when such materials are not used, as very often happens, the contact formed turns out to be nonideal. This is so because it is 
difficult to avoid the fact that very thin insulating layers and/or interface states may be present at the contact $[18,19]$. Hence a nonideal description of the contact should be used, with, for instance, a bias-dependent relation for the barrier height which would include the effects of the insulating layer and of the interface states. Such contacts have been described in detail in Refs. $[7,8]$. In particular, it has been shown that for this nonideal contact there is no simple description directly using equations such as Eqs. (11) or (12), for contact nonstationary effects induced by interface sates (not present for ideal MS contacts) introduce additional terms not present in Eq. (11). These terms could be of importance when describing naturally nonstationary phenomena such as the Gunn effect with nonideal contacts, and will be considered in future works.

Equation (12) and (13) can be rewritten in terms of the electric field by using the Poisson equation to eliminate the electron density from them:

$$
\frac{\partial \widetilde{E}}{\partial \widetilde{x}}(i, \widetilde{t})=\frac{e}{\varepsilon}\left(\tilde{n_{0}}-N_{C} e^{-e \beta \phi_{b}^{i} \pm \frac{J_{n}(i, \widetilde{t})}{\frac{\lambda_{i}}{N_{C}}}}\right) .
$$

Here the upper (lower) sign holds for $i=0[i=\widetilde{L}]$, and $\varepsilon$ and $\widetilde{n_{0}}$ are the bulk semiconductor permitivity and its doping, respectively.

We have thus shown how our procedure allows us to derive explicit and precise expressions for the b.c. imposed by a given contact. A first important consequence of this method can be drawn directly from Eq. (14), which is simply a relation between the normal derivative of the electric field and the current density at the contact. Examining our derivation shows that this result is simply a consequence of the use of kinetic models to describe the exchange of carriers through the contact. Hence, one should expect that b.c.'s derived in this way, will result in relationships between the normal derivative of the electric field and the current density at the contact. It is easily seen that (if diffusion effects can be neglected) our b.c.'s can be transformed into Kroemer's-type contact current-field control characteristics [11] (see Sec. III). However, unlike previous models following Kroemer's approach $[3,4,12,31]$, our control characteristics are the result of a physically precise derivation, and therefore only parameters which are physically well defined appear in them. In particular, to use our control characteristics we do not have to invoke ad hoc assumptions involving new parameters such as the contact length [4,31].

To facilitate the analysis in the rest of the paper, it is convenient to rewrite Eq. (14) in dimensionless units. This greatly reduces the number of relevant parameters. Our dimensionless electric field $\mathcal{E}$, electron densities $n$, current densities $j(x, t)$, time $t$, and position $x$ are measured in units of $\widetilde{E_{0}}, \widetilde{n_{0}}, e \widetilde{n_{0}} \mu_{0} \widetilde{E_{0}}, \varepsilon /\left(e \mu_{0} \widetilde{n_{0}}\right)$, and $\varepsilon \widetilde{E_{0}} /\left(e \widetilde{n_{0}}\right)$, respectively [32]. In these equations, $\widetilde{E}_{0}$ and $\mu_{0}$ are an electric field and the zero-field electron mobility typical of the processes occurring in the bulk of the semiconductor (see below). Then Eq. (14) becomes

$$
\frac{\partial \mathcal{E}}{\partial x}(i, t)=\alpha_{i}\left[-i_{i} \pm j(i, t)\right]
$$

where we defined

$$
\begin{gathered}
\alpha_{i}=\frac{\mu_{0} \widetilde{E_{0}} N_{C}}{\lambda_{i}}, \\
i_{i}=-\alpha_{i}^{-1}\left(1-\frac{N_{C}}{\widetilde{n_{0}}} e^{-e \beta \phi_{b}^{i}}\right) .
\end{gathered}
$$

As mentioned above, here the upper (lower) sign refers to $i=0(i=L)$. It is worth noting that $\alpha_{i}$ is always a positive quantity (because $\lambda_{i}$ is), while $i_{i}$ does not have a definite sign: it depends basically on the value of the barrier height $\phi_{b}^{i}$ and on the doping value $\tilde{n_{0}}$.

It should be noted that there are important restrictions on the possible values of the contact current density which are due to the fact that in Eqs. (12) and (13) the electron density $n$ is a positive quantity:

$$
0 \leqslant \widetilde{n}(i, \widetilde{t})=N_{C} e^{-e \beta \phi_{b}^{i} \pm \frac{N_{C}}{\lambda_{i}}} J_{n}(i, \widetilde{t}),
$$

or, in dimensionless units,

$$
\pm j(i, t)<\alpha_{i}^{-1}+i_{i} \equiv j_{i}^{\mathrm{sat}} .
$$

[Equations (16) and (17) imply that $\alpha_{i}^{-1}+i_{i}=j_{i}^{\text {sat }}$ is always a positive quantity, equal to the maximum current density which the contact can provide.] These restrictions on the current are reminiscent of the rectifying properties of MS contacts. In practice, they only impose a real limitation for the case of true rectifying contacts (when one of the $j_{i}^{\text {sat }}$ is small). Otherwise, i.e., for large values of $j_{i}^{\text {sat }}$, an Ohmic contact is obtained which does not impose a real limitation on the current.

In order to analyze the influence of the derived b.c.'s on the Gunn instability, we will assume a sample formed by a certain semiconductor (able to display the Gunn effect) and by two MS contacts implemented on it. The resulting b.c.'s are

$$
\begin{gathered}
\frac{\partial \mathcal{E}}{\partial x}(0, t)=\alpha_{0}\left[j(0, t)-i_{0}\right], \\
\frac{\partial \mathcal{E}}{\partial x}(L, t)=-\alpha_{L}\left[i_{L}+j(L, t)\right] .
\end{gathered}
$$

As discussed above, for a given semiconductor, the values of the contact parameters may vary somewhat depending on the metal used in the contact and on the preparation procedures. For instance, $\alpha_{i}$ may vary two orders of magnitude, from about 0.3 to 33.4 , if we use the experimental values of Richardson's constant for GaAs reported in Ref. [27]. Similarly, the values of $i_{i}$ may also span two orders of magnitude, from about 0.03 to 4.01 , due to the variation of $\alpha_{i}$, if we fix the barrier height $\phi_{b} \approx 0.2 \mathrm{~V}$ (corresponding to $\mathrm{Al}$ [29]), and the donor density is $10^{14} \mathrm{~cm}^{-3}$. Thus there is a rather wide range of parameter values for the contacts, corresponding to a large variety of situations which will be described in this paper. 
Finally, the applied bias $V$, defined as $e V(t)$ $=\hat{F}_{m}(L, t)-\hat{F}_{m}(0, t)$, can be expressed as follows: $V(t)$ $=\int_{0}^{L} \mathcal{E}(x, t) d x+\left(\hat{\phi}_{b}^{0}-\hat{\phi}_{b}^{L}\right)$. In the previous expressions, $V$ and $\hat{\phi}_{b}^{i}$ are in units of $\varepsilon \widetilde{E}_{0}^{2} / e \widetilde{n_{0}}$, and $\hat{F}_{m}$ in units of $\varepsilon \widetilde{E}_{0}^{2} / \widetilde{n_{0}}$.

Very frequently the analysis of the Gunn effect under dc voltage bias is carried out by using the opposite sign for the electric field: $E=-\mathcal{E}$. Then the b.c.'s become

$$
\begin{aligned}
& \frac{\partial E}{\partial x}(0, t)=\alpha_{0}\left[i_{0}-j(0, t)\right], \\
& \frac{\partial E}{\partial x}(L, t)=\alpha_{L}\left[i_{L}+j(L, t)\right] .
\end{aligned}
$$

With these definitions, the dc voltage is $V=-\int_{0}^{L} E d x-\left(\hat{\phi}_{b}^{L}-\hat{\phi}_{b}^{0}\right)$. Instead of working with the voltage $V$, it is more convenient to use the average electric field on the semiconductor sample, $\phi=L^{-1} \int_{0}^{L} E(x, t) d x$, which is equal to $\phi=(1 / L)\left[-V+\left(\hat{\phi}_{b}^{0}-\hat{\phi}_{b}^{L}\right)\right]$. In what follows, negative voltages $V<0$ will be considered such as $\phi>0$. With these conventions, the carriers go from the cathode (injecting contact) at $x=0$ to the anode (receiving contact) at $x=L$.

\section{KROEMER'S MODEL AND ITS STATIONARY STATES}

\section{A. Kroemer's model}

The unipolar drift-diffusion model for the Gunn effect proposed by Kroemer [2,33] is generally accepted to provide a rather complete description of the main features of this effect. Yet it is simple enough to allow a very detailed asymptotic analysis; other important models such as Büttiker and Thomas's [34] incorporate more detailed physics, but their study is technically more demanding. In the dimensionless units described above, Kroemer's model is

$$
\begin{gathered}
\frac{\partial E}{\partial t}+v(E)\left(\frac{\partial E}{\partial x}+1\right)-\delta \frac{\partial^{2} E}{\partial x^{2}}=J, \\
\frac{1}{L} \int_{0}^{L} E(x, t) d x=\phi .
\end{gathered}
$$

Equation (23) is Ampère's law, which says that the sum of the displacement current and drift-diffusion current is equal to the total current density $J(t)$. It can be obtained by differentiating the Poisson equation $\partial E / \partial x=n-1$ with respect to time, substituting the charge continuity equation $\partial n / \partial t$ $+\partial j(x, t) / \partial x=0$ [the electron current density is of the driftdiffusion type: $j(x, t)=n v(E)-\delta \partial n / \partial x]$, and then integrating the result with respect to $x$. The electron velocity is assumed to be $N$ shaped, and for specific calculations we shall use [33]

$$
v(E)=E \frac{1+B E^{4}}{1+E^{4}}
$$

(it has a maximum $v_{M}>0$ at $E_{M}>0$ followed by a minimum $0<v_{m}<v_{M}$ at $\left.E_{m}>E_{M}\right)$. The electron difusivity $\delta$ is as- sumed to be constant. The results using other curves having the same shape are similar. If $v(E)$ does not reach a minimum but saturates instead as $E \rightarrow \infty$, not all the monopole and dipole waves which we have found occur. Thus we have chosen the velocity curve that yields the richest dynamical behavior. The behavior of Kroemer's model with saturating velocity will be commented upon in Sec. IV. The dc bias $\phi$ is the average electric field on the semiconductor sample. Equations (23) and (24) need to be solved with an appropriate initial field profile $E(x, 0)$ and subject to the following mixed boundary conditions resulting from substituting $j(x, t)=J(t)-\partial E / \partial t$ [from Eq. (23)] into Eqs. (21) and (22):

$$
\begin{aligned}
& \frac{\partial E}{\partial x}(0, t)=\alpha_{0}\left(i_{0}-J(t)+\frac{\partial E}{\partial t}(0, t)\right), \\
& \frac{\partial E}{\partial x}(L, t)=\alpha_{L}\left(i_{L}+J(t)-\frac{\partial E}{\partial t}(L, t)\right) .
\end{aligned}
$$

In what follows, $i_{i}$ will be assumed to be positive because physically interesting phenomena (including the usual Gunn effect mediated by high-field domains) are observed for these values of $i_{i}$, as will be seen in the following sections.

For typical $n$-type GaAs data, $\delta \ll 1$ and $L \gg 1$ [12]. In this limit, we shall find approximate solutions to the initial boundary value problem Eqs. (23)-(27) for $E(x, t)$ and $J(t)$. Strictly speaking, the simple asymptotic description that follows holds in the limit $L \rightarrow \infty$, even when $\delta=O(1)$ [15]. Assuming $\delta \ll 1$ simplifies the description of the traveling waves of the electric field in the semiconductor through the use of characteristic equations and shock waves [12,35,36].

To take advantage of this limit, we will use the following rescaled time and length:

$$
\epsilon=\frac{1}{L}, \quad s=\frac{t}{L}, \quad y=\frac{x}{L} .
$$

Then Eqs. (23) and (24) become

$$
\begin{aligned}
J-v(E)= & \epsilon\left(\frac{\partial E}{\partial s}+v(E) \frac{\partial E}{\partial y}\right)-\delta \epsilon^{2} \frac{\partial^{2} E}{\partial y^{2}}, \\
& \int_{0}^{1} E(y, s) d y=\phi .
\end{aligned}
$$

\section{B. Stationary states and their stability}

Before describing the Gunn effect in the present model, it is convenient to discuss how to construct stationary solutions of the model in the limit $\epsilon \ll 1$ and $\delta \ll 1$. [In the case $\delta=O(1)$ the procedure is slightly more complicated, and we shall omit the corresponding details; see Ref. [32]. In this section we shall analyze the stationary states of Kroemer's model in $n$-type GaAs $[2,33]$ under dc voltage bias with the new boundary conditions, Eqs. (21) and (22). Our work is based upon previous asymptotic and numerical studies of this and related models [12-15].

In this asymptotic limit, any stationary solution can be described as composed of outer and inner solutions: the outer bulk solution is a piecewise constant field profile valid everywhere except for two narrow boundary layers located at 


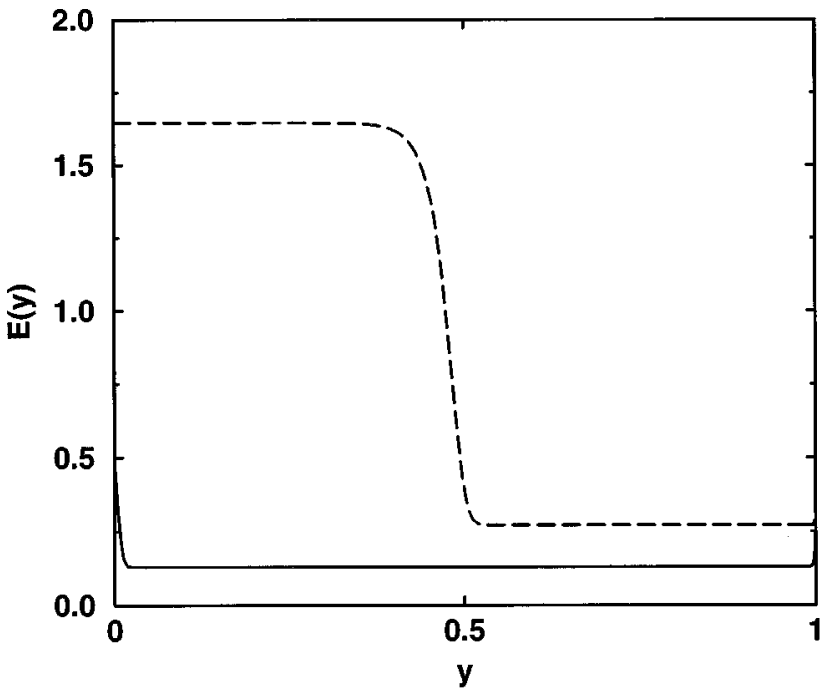

FIG. 1. Stationary electric-field profiles, showing the piecewise character of the solutions. The dashed line corresponds to a steplike stationary solution. Narrow boundary layers are present at the contacts.

the contacts and, for particular values of the current density, a narrow transition layer somewhere in the middle of the sample (see Fig. 1 and the explanation below). First of all, if we ignore inner solutions, the stationary state solves the equations

$$
\begin{gathered}
v(E)-J=O(\epsilon), \\
E=\phi+O(\epsilon),
\end{gathered}
$$

except for particular values of $J$ which will be specified below. These equations result from retaining only order-1 terms in Eqs. (29) and (30), and assuming $E(y)=$ const. Then for those values of $\phi$ such that the outer solution Eq. (31) is compatible with the boundary conditions, we have $J=v(\phi)+O(\epsilon)$. Let us denote by $E_{1}(J)<E_{2}(J)<E_{3}(J)$ the three zeros of $v(E)-J\left[E_{2}(J)\right.$ is unstable]. Then the outer (bulk) field profile will be $E(y)=E_{i}, i=1$ and 3, depending on the value of the bias $\phi$.

At $y=0$ and 1 there are boundary layers, which we will call injecting and receiving layers, respectively. $E(y ; J)$, the field at the injecting boundary layer of width $O(\epsilon)$ at $y=0$, obeys [we ignore the $O(\delta)$ diffusive term]

$$
\begin{gathered}
\epsilon \frac{\partial E}{\partial y}=\frac{J}{v(E)}-1, \quad x>0, \\
\epsilon \frac{\partial E}{\partial y}(0 ; J)=\alpha_{0}\left(i_{0}-J\right) .
\end{gathered}
$$

The analysis of Eqs. (32) and (33) is more easily carried out if we express the derivative b.c.'s Eq. (33) in terms of a b.c. for the electric field at the contact, $E(0 ; J)$. We can obtain $E(0 ; J)$ from Eq. (33) by using Eq. (32) to eliminate $\partial E(0 ; J) / \partial y$. The result is that $E=E(0 ; J)$ solves

$$
j_{c}(E)=J
$$

where

$$
j_{c}(E)=\frac{\left(1+\alpha_{0} i_{0}\right) v(E)}{1+\alpha_{0} v(E)} .
$$

Notice that the contact curve $j_{c}(E)$ has the same extrema as the velocity curve $v(E)$ and saturates for high electric fields to the value $j_{0}^{\text {sat }}$, defined in Eq. (18). Kroemer's contact characteristic for shallow-barrier metal-semiconductor contacts, presented in Ref. [33], corresponds to a particular case of our model in which the electrons in the metal are assumed to be in equilibrium with those of the semiconductor near the contact. For this case, one would take $\alpha_{0} \rightarrow 0$ with $\left|\alpha_{0} i_{0}\right|<\infty$, so that $j_{c}(E)$ would be then proportional to $v(E)$. In contradistinction with Kroemer's contact characteristic, the general curve $j_{c}(E)$ may intersect the bulk velocity curve $v(E)$. The main difference between these two cases is that a Gunn effect mediated by charge dipole waves is seen only if $j_{c}(E)$ intersects the bulk velocity curve $v(E)$. If Eq. (34) has a solution, Eq. (32) indicates that $E(y ; J)$ approaches one of the solutions of Eq. (31) as we leave the boundary layer. The boundary layer at the receiving contact $y=1$ is a much narrower diffusive boundary layer of width $O(\epsilon \delta)$. The field there is [32]

$$
\begin{gathered}
\frac{\partial E}{\partial \eta}=\int_{E}^{E_{i}} v(E) d E \\
\frac{1-y}{\epsilon \delta} \equiv \eta=\int_{E_{L}}^{E(\eta)} \frac{d F}{\int_{F}^{E_{i}} v(E) d E}
\end{gathered}
$$

where

$$
\alpha_{L}\left(i_{L}+J\right)=-\frac{1}{\delta} \int_{E_{L}}^{E_{i}} v(E) d E
$$

( $i=1$ and 3) whenever Eq. (38) has a solution $E_{L}$.

The idea now is to fix $J$ and to discuss for which values of $J$ the above construction yields a stationary solution. Additionally, its stability will be considered. Clearly we may distinguish different cases according to the values of the contact parameters $\left(i_{i}, \alpha_{i}\right), i=0, L$. In what follows we shall assume for the sake of simplicity that the boundary layer equations at the receiving contact, Eqs. (37) and (38), always have a solution, and hence only the parameters of the cathode $\left(i_{0}\right.$, $\alpha_{0}$ ) need to be considered.

The general situation encountered when constructing the stationary solutions is the following. For each value of $J$, there are one or three values of the contact electric field at $x=0$, which are solutions of Eq. (34). We shall denote these field values by $E_{c i}(J)$, with $E_{c 1}(J)<E_{c 2}(J)<E_{c 3}(J)$. Then the field profile in the injecting boundary layer is a monotonic solution of Eq. (32), which joins $E_{c i}(J)(i=1,2$, and $3)$ to one of the solutions of $J=v(E)$ (outer solution). Furthermore, the outer solution may be a constant field profile given by $E(y)=E_{l}(J)(l=1$ and 3$)$ which extends to the end of the sample, where a narrow receiving boundary layer exists (see Fig. 1). For such an electric-field profile, the bias is $\phi \approx E_{i}(J)$. The corresponding $J-\phi$ characteristics satisfy $J \approx v(\phi)$. By this construction, we identify the portions of the $J-\phi$ characteristics which follow the first or third branch of $v(E)$ (see the details below). Other portions of the $J-\phi$ 


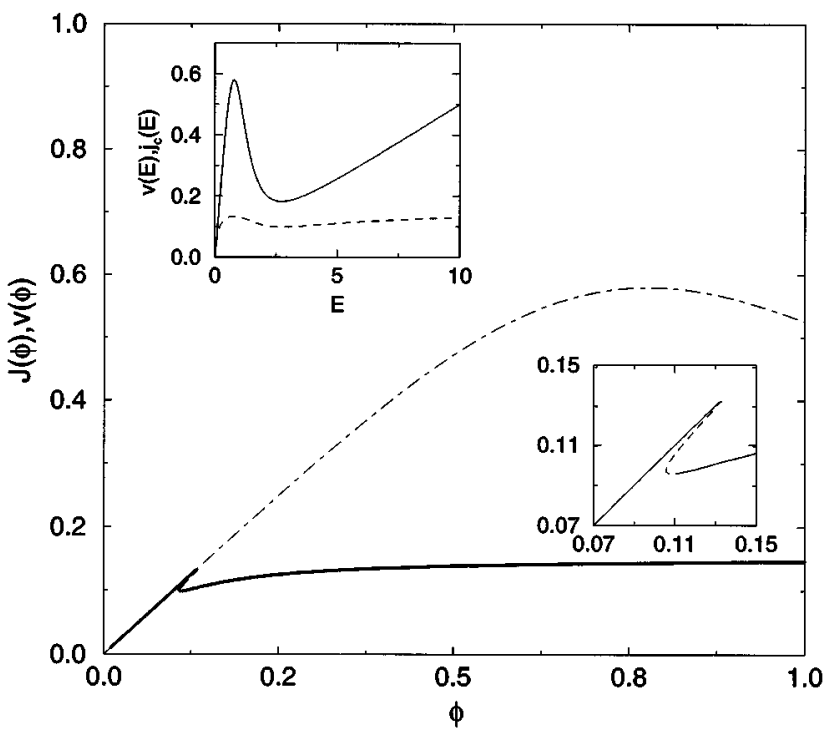

FIG. 2. Stationary current-voltage characteristics, for $L=500$, $i_{0}=0.048$, and $\alpha_{0}=9$, for which $j_{c m}<j_{c M}<j_{0}^{\text {sat }}<v_{m}<v_{M}$, showing bistability for biases $E_{1}\left(j_{c} m\right)<\phi<E_{1}\left(j_{c} M\right)$. The dashed line corresponds to the unstable solutions with $E(0 ; J)=E_{c 2}(J)$. For comparison the $v(\phi)$ curve is also plotted (dotted-dashed line). Insets: At the bottom, enlargement of the bistable region. On top, contact characteristics $j_{c}(E)$ (dashed line) and velocity $v(E)$ (continuous line) curves for this case.

characteristics are flat, with $J=J_{f}$ for certain constant values of the current. The corresponding outer field profile is steplike, with $E=E_{2}\left(J_{f}\right)=E_{c 2}\left(J_{f}\right)$ if $0<y<\Delta Y$ and $E=E_{i}\left(J_{f}\right)$ with $i=1$ and 3 if $\Delta Y<y<1$ (see Fig. 1). $\Delta Y$ is chosen so as to satisfy the bias condition $\phi \approx E_{2}\left(J_{f}\right) \Delta Y+(1-\Delta Y) E_{i}\left(J_{f}\right)$. The flat part of the $J-\phi$ characteristics corresponds to a bias range $E_{1}\left(J_{f}\right)<\phi<E_{3}\left(J_{f}\right)$ (see the details below). Finally, when $J$ is near the value $j_{0}^{\text {sat }}$, the field at the injecting contact is very large, the contact region is almost depleted of electrons, and its extension $y=\epsilon\left[E_{c 3}(J)-E_{i}(J)\right](i=1$ and 3) may be comparable to the length of the sample. Assuming the extension of the depletion layer at the injecting contact is less than the sample length, the corresponding bias is $\phi \approx \frac{1}{2} \epsilon\left[E_{c 3}(J)-E_{i}(J)\right]^{2}+E_{i}(J)(i=1$ and 3$)$. The characteristics tends to saturate at $j_{0}^{\text {sat }}$.

Following this general scheme, different possibilities may be distinguished according to the relative values of $j_{\mathrm{cm}}$ $<j_{c M}<j_{0}^{\text {sat }}$ with respect to $v_{m}<v_{M}$. Here $j_{c k}=(1$ $\left.+\alpha_{0} i_{0}\right) v_{k} /\left(1+\alpha_{0} v_{k}\right)$ and $v_{k}$, with $k=m, M$, refer to the minimum $(k=m)$ and maximum $(k=M)$ of the contact $j_{c}(E)$ and velocity $v(E)$ curves, respectively. We now discuss the different cases which appear for our velocity curve.

$$
\text { 1. } j_{c m}<j_{c M}<j_{0}^{\text {sat }}<v_{m}<v_{M}
$$

In this case (see Fig. 2), for $0<J<J_{c M}$ we have a class of solutions joining $E_{c 1}(J)$ and $E_{1}(J)$, with voltage $\phi \approx E_{1}(J)$. For $0<\phi<E_{1}\left(j_{c M}\right)$ the curve $J-\phi$ then follows the first branch of $v(E)$. Furthermore, a second class of solutions joining $E_{c 3}(J)$ and $E_{1}(J)$, will exist for $j_{c m}<J<j_{0}^{\text {sat }}$. In this case for $J$ not near $j_{0}^{\text {sat }}$, the voltage is given by $\phi \approx E_{1}(J)$, and for $J$ near $j_{0}^{\text {sat }}$ it is by

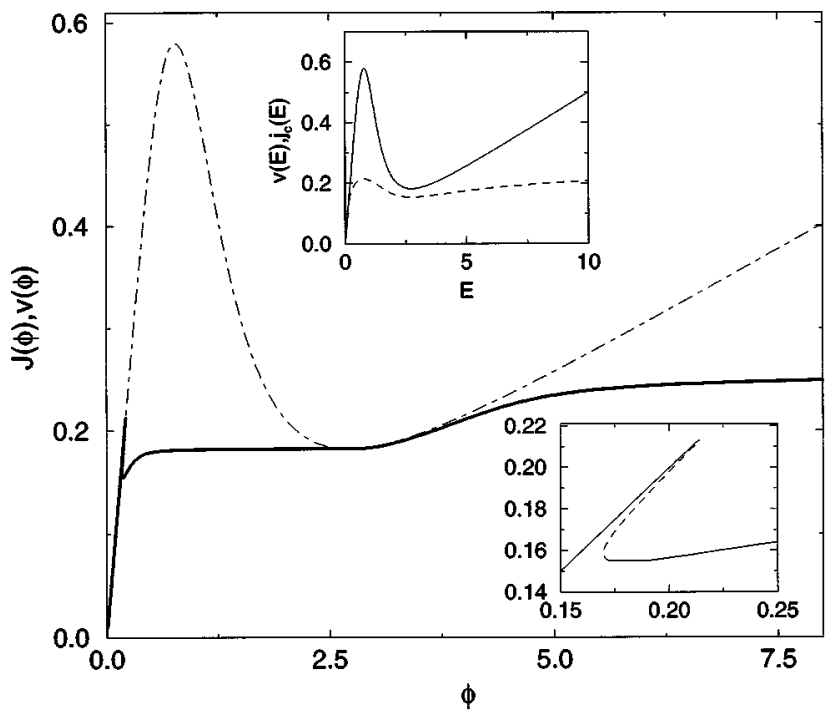

FIG. 3. Stationary current-voltage characteristics, for $L=500$, $i_{0}=0.135$, and $\alpha_{0}=8$, for which $j_{c m}<v_{m}<j_{c M}<j_{0}^{\text {sat }}<v_{M}$, showing bistability for biases $E_{1}\left(j_{c m}\right)<\phi<E_{1}\left(j_{c M}\right)$. The dashed line corresponds to the unstable solutions with $E(0 ; J)=E_{c 2}(J)$. The flat portion of the curve corresponds to $J=v_{m}$. A Gunn effect mediated by moving depletion charge monopoles is expected on a bias range which is a subinterval of $E_{M}<\phi<E_{m}$. For comparison the $v(\phi)$ curve is also plotted (dotted-dashed line). Insets: At the bottom, enlargement of the bistable region. On top, contact characteristics $j_{c}(E)$ (dashed line) and velocity $v(E)$ (continuous line) curves for this case.

$\phi \approx \frac{1}{2} \epsilon\left[E_{c 3}(J)-E_{1}(J)\right]^{2}+E_{1}(J)$. Then in the characteristics the third branch starting at $\phi \approx E_{1}\left(j_{c m}\right)$ follows the first branch of $v(E)$ at the beginning, until it tends to saturate to $j_{0}^{\text {sat }}$ for larger voltages (see Fig. 2). Joining these two classes of solutions, there exists a third class for $j_{c m}<J<j_{c M}$, which joins $E_{c 2}(J)$ to $E_{1}(J)$, with $\phi \approx E_{1}(J)$. These solutions are unstable, and they give rise to the second branch in the characteristics, Fig. 2, which also tend to follow the first branch of $v(E)$. Note that for voltages $E_{1}\left(j_{c} m\right)$ $<\phi<E_{1}\left(j_{c} M\right)$, the two classes of stable stationary solutions coexist (see inset at the bottom of Fig. 2). Hysteresis between them is then possible.

$$
\text { 2. } j_{c m}<\boldsymbol{v}_{m}<j_{c M}<j_{0}^{\text {sat }}<\boldsymbol{v}_{M}
$$

In this case (see Fig. 3), the description is very similar to the previous case, except on what concerns the third branch of the $J-\phi$ characteristics. Now this branch is composed of two types of solutions: (i) for $j_{c m}<J<v_{m}$, there is a class of solutions joining $E_{c 3}(J)$ and $E_{1}(J)$. Most of the time one has $\phi \approx E_{1}(J)$, except for $J$ near $v_{m}$ that the solution is steplike with $\phi \approx E_{m} \Delta Y+(1-\Delta Y) E_{1}\left(v_{m}\right)$. We expect that these solutions become unstable on a bias range which is a subinterval of $E_{M}<\phi<E_{m}[37,38]$. Then a Gunn effect mediated by moving charge monopoles (which are charge depletion layers) might appear (see the companion paper [16]). For $v_{m}<J<j_{0}^{\text {sat }}$, there is a class of solution joining $E_{c 3}(J)$ and $E_{3}(J)$, with $\phi \approx E_{3}(J)$ for $J$ not near $j_{0}^{\text {sat }}$ and $\phi \approx \frac{1}{2} \epsilon\left[E_{c 3}(J)-E_{3}(J)\right]^{2}+E_{3}(J)$ for $J$ near $j_{0}^{\text {sat }}$. Then the third branch of the $J-\phi$ curve starts following the first branch 


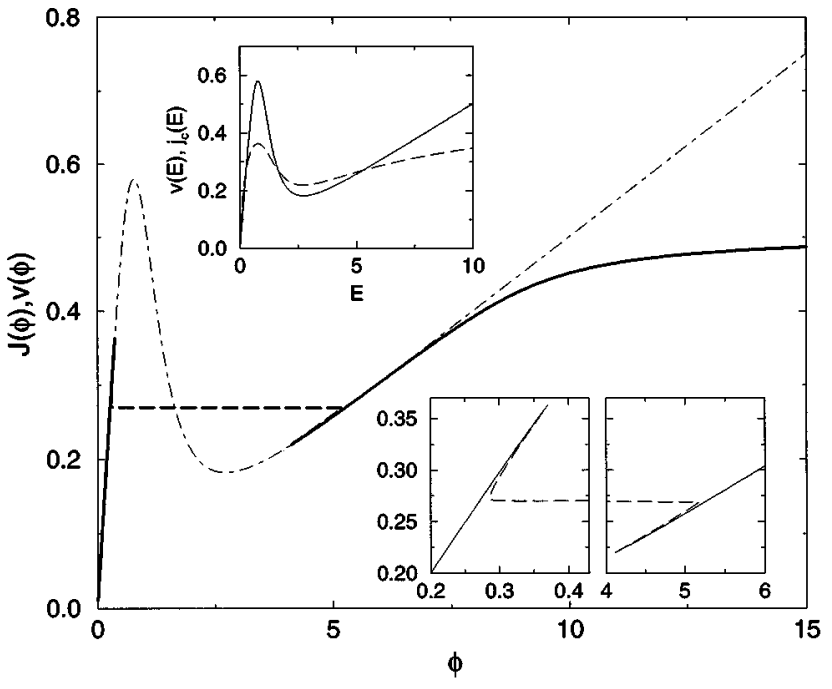

FIG. 4. Stationary current-voltage characteristic for $L=500$, $i_{0}=0.27$, and $\alpha_{0}=4$, for which $v_{m}<j_{c m}<j_{c M}<j_{0}^{\text {sat }}<v_{M}$, showing the unstable stationary solutions (dashed line) with $E(0 ; J)=E_{c 2}(J)$. The flat portion of the curve corresponds to $J=i_{0}$. Note that no stable stationary solution exists for $E_{1}\left(j_{c M}\right)<\phi<E_{3}\left(j_{c m}\right)$. Then a Gunn effect mediated by moving charge dipoles is expected. For comparison the $v(\phi)$ curve is also plotted (dotted-dashed line). Insets: At the bottom, enlargement of the unstable region. On top, contact characteristics $j_{c}(E)$ (dashedline) and velocity $v(E)$ (continuous line) curves for this case.

of the $v(E)$ curve for $E_{1}\left(j_{c m}\right)<\phi<E_{1}\left(v_{m}\right)$, then it presents a flat region for $E_{1}\left(v_{m}\right)<\phi<E_{m}$ with $J=v_{m}$, corresponding to the steplike solutions, and finally a region for $E_{m}<\phi$ which starts following the third branch of the $v(E)$ curve and tends to saturate to $j_{0}^{\text {sat }}$ for larger voltages. Note the presence again of bistability for voltages $E_{1}\left(j_{c m}\right)$ $<\phi<E_{1}\left(j_{c M}\right)$ (the inset at the bottom of Fig. 3) and hence the possibility of hysteresis. A similar situation to the one depicted above would appear for $j_{c m}<j_{c M}<v_{m}<j_{0}^{\text {sat }}<v_{M}$.

$$
\text { 3. } v_{m}<j_{c m}<j_{c M}<j_{0}^{\mathrm{sat}}<v_{M}
$$

The main difference for this case with respect to the previous ones, relies on the second and third branches (see Fig. 4). Now the third branch of the $J-\phi$ curve involves one single class of solutions joining $E_{c 3}(J)$ and $E_{3}(J)$, with voltages $\phi \approx E_{3}(J)$ for $J$ not near $j_{0}^{\text {sat }}$, and $\phi \approx \frac{1}{2} \epsilon\left[E_{c 3}(J)-E_{3}(J)\right]^{2}+E_{3}(J)$ for $J$ near $j_{0}^{\text {sat }}$. The second (unstable) branch is formed of two classes of solutions: one class for $i_{0}<J<j_{c M}$ starting at $E_{c 2}(J)$ and ending at $E_{1}(J)$, and another class for $j_{c m}<J<i_{0}$, starting at $E_{c 2}(J)$ and ending at $E_{3}(J)$. For $J \approx i_{0}$, these solutions are steplike, with the voltage given through $\phi \approx E_{c 2}\left(i_{0}\right) \Delta Y+(1$ $-\Delta Y) E_{i}(J)$, with $i=1$ and 3 depending on the class of solutions considered. The (unstable) branch in the $J-\phi$ curve then starts following the first branch of the $v(E)$ curve for $E_{1}\left(j_{c M}\right)<\phi<E_{1}\left(i_{0}\right)$; then it presents a flat portion for $E_{1}\left(i_{0}\right)<\phi<E_{3}\left(i_{0}\right)$ with $J \approx i_{0}$, and it ends following the third branch of the $v(E)$ curve for $E_{3}\left(j_{c m}\right)<\phi<E_{3}\left(i_{0}\right)$. Note that for voltages $E_{1}\left(j_{c M}\right)<\phi<E_{3}\left(j_{c m}\right)$ there is no stable stationary solution (see the inset at the bottom of Fig. 4). Thus we expect that the usual Gunn effect (mediated by

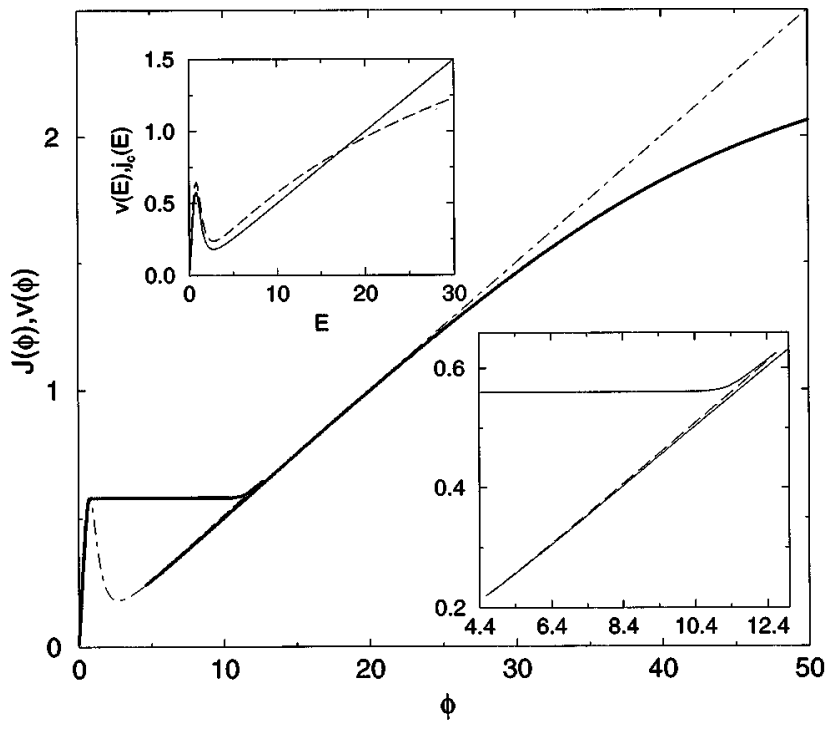

FIG. 5. Stationary current-voltage characteristic for $L=500$, $i_{0}=0.87$, and $\alpha_{0}=0.5$, for which $v_{m}<j_{c m}<v_{M}<j_{c M}<j_{0}^{\text {sat }}$, showing bistability for biases $E_{3}\left(j_{c m}\right)<\phi<E_{3}\left(j_{c M}\right)$. The dashed line corresponds to the unstable stationary solutions with $E(0 ; J)=E_{c 2}(J)$. The flat portion of the curve corresponds to $J=v_{m}$. A Gunn effect mediated by moving depletion charge monopoles is expected on a bias range which is a subinterval of $E_{M}<\phi<E_{m}$. For comparison the $v(\phi)$ is also plotted (dotteddashed line). Insets: At the bottom, enlargement of the bistable region. On top, contact characteristics $j_{c}(E)$ (dashed line) and velocity $v(E)$ (continuous line) curves for this case.

moving charge dipoles) will be present for these values of the bias (see the companion paper [16]). A similar situation appears for $v_{m}<j_{c m}<j_{c M}<v_{M}<j_{0}^{\text {sat }}$.

\section{4. $v_{m}<j_{c m}<v_{M}<j_{c M}<j_{0}^{\text {sat }}$}

In this case (see Fig. 5), the third branch of the $J-\phi$ characteristics is described as in the previous case. The first branch is composed of two types of solutions: one class, for $0<J<v_{M}$, joining $E_{c 1}(J)$ and $E_{1}(J)$, and the other, for $v_{M}<J<j_{c M}$, joining $E_{c 1}(J)$ and $E_{3}(J)$. For the first type of solutions, one has $\phi \approx E_{1}(J)$, except for $J \approx v_{M}$ that $\phi=E_{M} \Delta Y+(1-\Delta Y) E_{3}\left(v_{M}\right)$. These steplike solutions are expected to become unstable in a subinterval of $E_{M}<\phi<E_{m}$ $[37,38]$. Then a Gunn effect mediated by moving charge monopoles (which are charge accumulation layers) might appear (see the companion paper [16]). Thus the first branch starts following the first branch of the $v(E)$ curve for $0<\phi<E_{M} ;$ then it presents a flat portion for $E_{M}<\phi<E_{3}\left(v_{M}\right)$, with $J=v_{M}$, and ends following the third branch of the $v(E)$ for $E_{3}\left(v_{M}\right)<\phi<E_{3}\left(j_{c M}\right)$. The second (unstable) branch of the $J-\phi$ curve is formed by a class of solutions that starts at $E_{c 2}(J)$ and ends at $E_{3}(J)$, with $\phi \approx E_{3}(J)$. Then this branch follows the third branch of the $v(E)$ curve, for $E_{3}\left(j_{c m}\right)<\phi<E_{3}\left(j_{c M}\right)$. Note that, for this range of bias, two stationary stable solution coexists (see the inset at the bottom of Fig. 5) and hysteresis may appear.

\section{Phase diagram}

By collecting the information obtained in the previous subsections, the phase diagram describing the different be- 


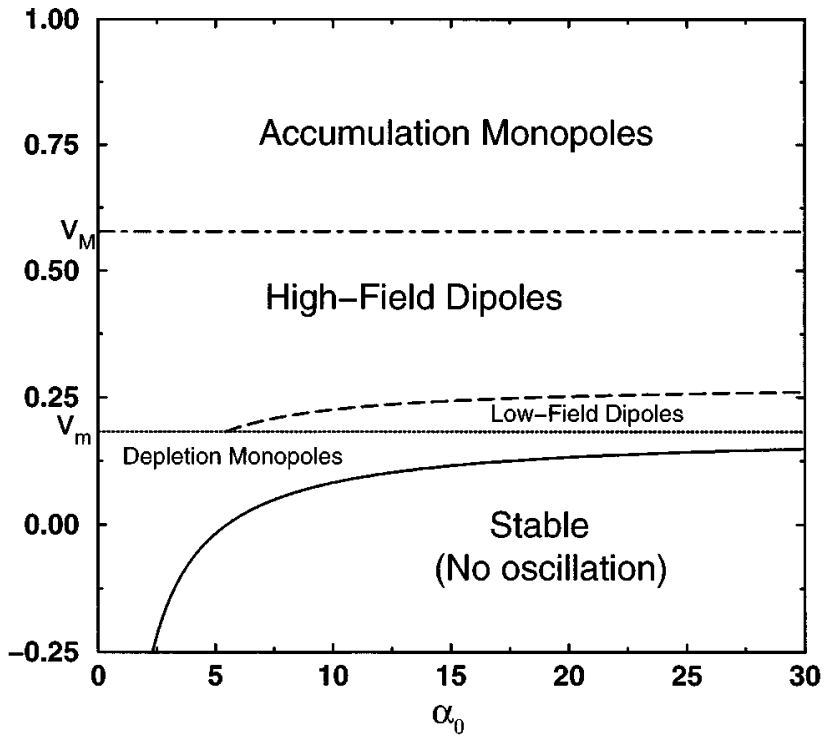

FIG. 6. Phase diagram, showing the diversity of Gunn oscillations that may appear depending on the values of the injecting contact parameters $i_{0}$ and $\alpha_{0}$. The different separatrices correspond to $j_{0}^{\text {sat }}=v_{m}$ (continuous line), $j_{c m}=v_{m}$ (dotted line), and $j_{c M}=v_{M}$ (dotted-dashed line). Also depicted the separation between low- and high-field charge dipoles, discussed in the companion paper (dashed line).

haviors of the system can be sketched, in terms of the injecting contact parameters $i_{0}$ and $\alpha_{0}$, Fig. 6. Stable, nonoscillatory stationary solutions are expected for values of $i_{0}$ and $\alpha_{0}$ such as $j_{0}^{\text {sat }}<v_{m}$, where $j_{0}^{\text {sat }}=\alpha_{0}^{-1}+i_{0}$. Otherwise, some kind of oscillatory solution should be found. Charge accumulation monopoles appear for $j_{c M}>v_{M}$ (or equivalently for $i_{0}>v_{M}$ ), charge dipoles for $v_{m}<j_{c m}<j_{c M}<v_{M}$ (that is, for $v_{m}<i_{0}<v_{M}$ ), and charge depletion monopoles for $j_{0}^{\text {sat }}>v_{m}$ with $j_{c m}<v_{m}\left(i_{0}<v_{m}\right)$. For completeness, the separation between low- and high-field dipoles, discussed in the companion paper [16], has also been depicted. It is worth noting that this rich phenomenology of oscillatory states appears just by changing the value of the contact parameters. This fact should be taken into account in analyzing the Gunn effect in real systems, where, as mentioned above, a wide range of values for the contact parameters, depending on the metal used and preparation procedures, may appear.

\section{DISCUSSION}

We have presented a general formulation for the derivation of the boundary conditions imposed by metalsemiconductor contacts on semiconductor systems. According to this general formulation, the appropriate boundary conditions for ideal metal-semiconductor contacts are linear relations between the normal derivative of the electric field at the contacts and the electron current there. For the classical unipolar drift-diffusion Kroemer's model of the Gunn effect, these boundary conditions are of mixed type. In this paper, we have investigated how the boundary conditions for ideal metal-semiconductor contacts affect the stationary solutions of the Kroemer model, and their stability. Depending on the values of the contact parameters, bistability and hysteresis may appear. Moreover, for some range of parameters, no stable stationary solution is expected to occur. In those parameter ranges we expect to find the Gunn effect. Numerical simulations show that different types of Gunn effects appear, mediated by a variety of waves: (i) charge monopole accumulation wave fronts, (ii) monopole depletion wave fronts, or (iii) charge dipole waves (high and low electric field domains). Why these types of Gunn effects appear in the simulations will be explained by the asymptotic theory of the companion paper [16]. It suffices to say that without this theory we would have missed significant possible instabilities. For example, (ii) seems to have been missed by earlier workers, in spite of past extensive simulations of Kroemer's model [3]. With our boundary conditions, the previously described types of Gunn effect are found in the following ranges of dimensionless critical contact currents: (i) $j_{c M}>v_{M}$, (ii) $j_{c m}<v_{m}$ and $j_{0}^{\text {sat }}>v_{m}$, and (iii) $v_{m}<j_{c m}$ $<j_{c M}<v_{M}$. Here $j_{c M}=j_{c}\left(E_{M}\right), j_{c m}=j_{c}\left(E_{m}\right)$, and $j_{0}^{\text {sat }}$ are the critical currents, and $v_{m}$ and $v_{M}$ are the minimum and maximum values of the electron drift velocity $v(E), E>0$. When we want to characterize experimental samples displaying the Gunn effect, it is important to bear in mind the great influence of the contact parameters on the type of wave mediating the Gunn effect. A wide range of values for these contact parameters may be obtained depending on the type of metal used or on the contact preparation procedure followed.

$\mathrm{N}$-shaped velocity curves occur naturally in recently observed self-sustained oscillations in weakly coupled $n$-type doped GaAs/AlAs superlattices (see Ref. [39] for the most complete data so far). In these superlattices there is strong indirect evidence of a Gunn effect mediated by charge accumulation monopoles through photocurrent and photoluminescence measurements [40]. It is hard to say at this point which other possibilities of those found in our analysis might be realizable in these systems. An important issue to be decided is the form of the boundary conditions. Our analysis needs to be modified in order to be extended to these systems, as quantum tunneling plays an essential role in the injection of carriers through contact regions.

The most used velocity curves $v(E)$ for the classical Gunn effect in bulk $n$-type GaAs lack the third branch after $v_{m}$. The reason is that avalanche breakdown appears at electric fields smaller than $E_{m}$. The avalanche field is smaller for the longer samples needed to observe the Gunn effect, and this precludes reaching the high fields on the third branch of $v(E)$. Then low-field dipole domains and charge depletion monopoles are not observed in the usual bulk samples or in strongly coupled superlattices with wide minibands, which are analogous to them [34].

\section{ACKNOWLEDGMENTS}

We thank Dr. M. Bergmann for fruitful conversations, and acknowledge financial support from the Spanish DGICYT through Grant Nos. PB94-0375 and PB95-0881, and from the EC Human Capital and Mobility Program Contract No. ERBCHRXCT930413. One of us (G.G.) acknowledges support by the Generalitat de Catalunya.

\section{APPENDIX: DERIVATION OF EQ. (11) BY MEANS OF SRH STATISTICS}

Let us consider the elementary kinetic process $q^{m} \rightleftharpoons q^{n}$ describing the charge transport through the junction. By as- 
suming the validity of the SRH statistics to describe this process, the following expression for its kinetic rate, $J_{n}$, can be obtained [23]:

$$
\begin{aligned}
J_{n}= & \int d E_{n} \int d E_{m} D_{m}\left(E_{m}\right) D_{n}\left(E_{n}\right)\left[f_{m}\left(E_{m}\right)\left(1-f_{n}\left(E_{n}\right)\right)\right. \\
& \left.\times \gamma_{m n}\left(E_{m}, E_{n}\right)-f_{n}\left(E_{n}\right)\left(1-f_{m}\left(E_{m}\right)\right) \gamma_{n m}\left(E_{m}, E_{n}\right)\right],
\end{aligned}
$$

where $D_{a}\left(E_{a}\right), a=n, m$, is the density of states of system $a$, $f_{a}\left(E_{a}\right)$ its occupation function, given through the FermiDirac distribution $f_{a}\left(E_{a}\right)=\left(1+e^{\beta\left(E_{a}-E_{F_{a}}\right)}\right)^{-1}$, with $E_{F, a}$ the corresponding Fermi level. $\gamma_{m n}\left(E_{m}, E_{n}\right)\left[\gamma_{n m}\left(E_{m}, E_{n}\right)\right]$ is the probability per unit time for the transition between states of energy $E_{m}$ and $E_{n}\left(E_{n}\right.$ and $\left.E_{m}\right)$. At equilibrium, we must have $J_{n}=0$ and $F_{m}=F_{n}$, with $F_{a}=E_{F, a}-e V_{a}$ being the corresponding quasi-Fermi levels. This implies $E_{F, n}-E_{F, m}$ $=e\left(V_{n}-V_{m}\right)=0$ (using the assumed continuity of the electric potential at the contact). These equations follow from Eq. (A1) if the latter is supplemented with the following detailed balance relation:

$$
\gamma_{m n}\left(E_{m}, E_{n}\right)=\gamma_{n m}\left(E_{m}, E_{n}\right) e^{\beta\left(E_{m}-E_{n}\right)} .
$$

A term $\beta$ ( $\left.V_{n}-V_{m}\right)$ has to be added to the argument of the exponential in Eq. (A2) if $V_{n} \neq V_{m}$; see Ref. [8] for a more general case. We now substitute Eq. (A2) into Eq. (A1) and use the equations

$$
E_{C}=E_{C}^{0}-e V_{n}, \quad e \phi_{b}^{0}=E_{C}-F_{m}
$$

( $E_{C}^{0}$ is the bottom of the semiconductor conduction band and

$e \phi_{b}^{0}$ is the height of the contact barrier). After straightforward manipulations, we derive Eq. (11), in which the transition coefficient $\lambda_{0}$ is

$$
\begin{aligned}
\lambda_{0}= & \int d E_{n} \int d E_{m} D_{n}\left(E_{n}\right) D_{m}\left(E_{m}\right)\left(1-f_{n}\left(E_{n}\right)\right) \\
& \times\left(1-f_{m}\left(E_{m}\right)\right) \gamma_{n m}\left(E_{m}, E_{n}\right) e^{\beta\left(E_{C}^{0}-E_{n}\right)} .
\end{aligned}
$$

When the semiconductor is nondegenerate, we may approximate $1-f_{n}\left(E_{n}\right) \approx 1$, whereas for a metal we may approximate $f_{m}\left(E_{m}\right)$ by its equilibrium value. Then, for this case, $\lambda_{0}$ is a function of $T$ only.
[1] J. B. Gunn, Solid State Commun. 1, 88 (1963).

[2] H. Kroemer, in Topics in Solid State and Quantum Electronics, edited by W. D. Hershberger (Wiley, New York, 1972), p. 20.

[3] M. P. Shaw, H. L. Grubin, and P. R. Solomon, The GunnHilsum Effect (Academic, New York, 1979).

[4] M. P. Shaw, V. V. Mitin, E. Schöll, and H. L. Grubin, The Physics of Instabilities in Solid State Electron Devices (Plenum, New York, 1992).

[5] S.R. de Groot and P.Mazur, Non-Equilibrium Thermodynamics (Dover, New York, 1984).

[6] G. Gomila and J. M. Rubí, Physica A 243, 251 (1997).

[7] G. Gomila, A. Pérez-Madrid, and J. M. Rubí, Physica A 233, 208 (1996).

[8] G. Gomila and J. M. Rubí, J. Appl. Phys. 81, 2674 (1997).

[9] K.-I. Nakamura, J. Phys. Soc. Jpn. 38, 46 (1975).

[10] P. Szmolyan, Physica D 39, 393 (1989).

[11] H. Kroemer, IEEE Trans. Electron Devices ED-15, 819 (1968).

[12] F. J. Higuera and L. L. Bonilla, Physica D 57, 161 (1992).

[13] L. L. Bonilla, M. Kindelan, M. Moscoso, and S. Venakides, SIAM (Soc. Ind. Appl. Math.) J. Appl. Math. (to be published).

[14] L. L. Bonilla, P. J. Hernando, M. A. Herrero, M. Kindelan, and J. J. L. Velázquez, Physica D (to be published).

[15] L. L. Bonilla and I. R. Cantalapiedra, Phys. Rev. E (to be published).

[16] L. L. Bonilla, I. Cantalapiedra, G. Gomila, and J. M. Rubí, Phys. Rev. E (to be published).

[17] V. L. Rideout, Solid-State Electron. 18, 541 (1975).

[18] L. J. Brillson, Surf. Sci. 299/300, 909 (1994).

[19] W. Mönch, Surf. Sci. 299/300, 928 (1994).

[20] W. Shockley and W. T. Read, Phys. Rev. 87, 835 (1952).

[21] R. N. Hall, Phys. Rev. 87, 387 (1952).

[22] D. Bedeaux and P. Mazur, Physica A 82, 438 (1976).
[23] K. M. van Vliet, Phys. Rev. 110, 50 (1958).

[24] I. Pagonabarraga and J. M. Rubí, Physica A 188, 553 (1992).

[25] C. R. Crowell and S. M. Sze, Solid-State Electron. 9, 1035 (1966).

[26] S. M. Sze, Physics of Semiconductor Devices, 2nd ed. (Wiley, New York, 1981).

[27] G. Eftekhari, Phys. Status Solidi A 140, 189 (1993).

[28] E. H. Rhoderick and R. H. Williams, Metal-Semiconductor Contacts, 2nd ed. (OSP, New York, 1987).

[29] R. E. Vituro, J. L. Shaw, C. Mailhiot, L. J. Brillson, N. Tache, J. McKinley, G. Margaritondo, J. M. Woodall, P. D. Kirchner, G. D. Petit, and S. L. Wright, Appl. Phys. Lett. 52, 2052 (1988).

[30] R. E. Vituro S. Chang, J. L. Shaw, C. Mailhiot, L. J. Brillson, A. Terrasi, Y. Hwu, G. Mangaritondo, P. D. Kirchner, and J. M. Woodall, J. Vac. Sci. Technol. B 7, 1007 (1989).

[31] K. F. Wu, J. Czekaj, and M. P. Shaw, J. Appl. Phys. 74, 315 (1993).

[32] L. L. Bonilla and F. J. Higuera, Physica D 52, 458 (1991).

[33] H. Kroemer, IEEE Trans. Electron Devices ED-13, 27 (1966).

[34] M. Büttiker and H. Thomas, Z. Phys. B 34, 301 (1979).

[35] B. W. Knight and G. A. Peterson, Phys. Rev. 147, 617 (1966).

[36] L. L. Bonilla, SIAM (Soc. Ind. Appl. Math) J. Appl. Math. 51, 727 (1991).

[37] L. L. Bonilla, F. J. Higuera, and S. Venakides, SIAM (Soc. Ind. Appl. Math.) J. Appl. Math. 54, 1521 (1994).

[38] L. L. Bonilla and F. J. Higuera, SIAM (Soc. Ind. Appl. Math.) J. Appl. Math. 55, 1625 (1995).

[39] J. Kastrup, R. Hey, K. H. Ploog, H. T. Grahn, L. L. Bonilla, M. Kindelan, M. Moscoso, A. Wacker, and J. Galán, Phys. Rev. B 55, 2476 (1997).

[40] L. L. Bonilla, in Nonlinear Dynamics and Pattern Formation in Semiconductors and Devices, edited by F.-J. Niedernostheide (Springer, Berlin, 1995), p. 1. 